\title{
Parallel Coupling of Ion Mobility Spectrometry and Ion Trap Mass Spectrometry for the Real- Time Alarm Triggering and Identification of Hazardous Chemical Leakages
}

Mei $\mathrm{Li}^{\dagger, \hbar,}$, Shuang Wang ${ }^{\dagger, \hbar, \&, \downarrow}$, Chuting $\mathrm{Xu}^{\dagger, \hbar, \S}$, Huiwen Ruan ${ }^{\dagger, \hbar,}$, Weiguo Wang $^{\dagger, \S, *}$, Chuang Chen ${ }^{\dagger, \S, *}$ and Haiyang $\mathrm{Li}^{\dagger, \S, *}$

${ }^{\dagger}$ CAS Key Laboratory of Separation Science for Analytical Chemistry, Dalian Institute of Chemical Physics, Chinese Academy of Sciences, Dalian 116023, China

University of Chinese Academy of Sciences, Beijing 100049, China

${ }^{\S}$ Dalian key Laboratory for Online Analytical Instrumentation, Dalian 116023, China

ZZhengzhou Tobacco Research Institute of CNTC, Zhengzhou, 450001, China

${ }^{*}$ Corresponding Authors. Haiyang Li, email: hli@dicp.ac.cn; Weiguo Wang, email: wwg1978@dicp.ac.cn; Chuang Chen, email: chenchuang@dicp.ac.cn 


\section{Table of Contents}

1. The Schematic Diagram of Pulse Injection Structure. ........................ S3

2. The Relative Position of Sampling Inlet of ITMS System to the Axle

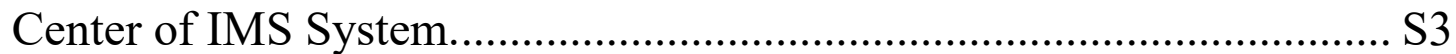




\section{The Schematic Diagram of Pulse Injection Structure.}

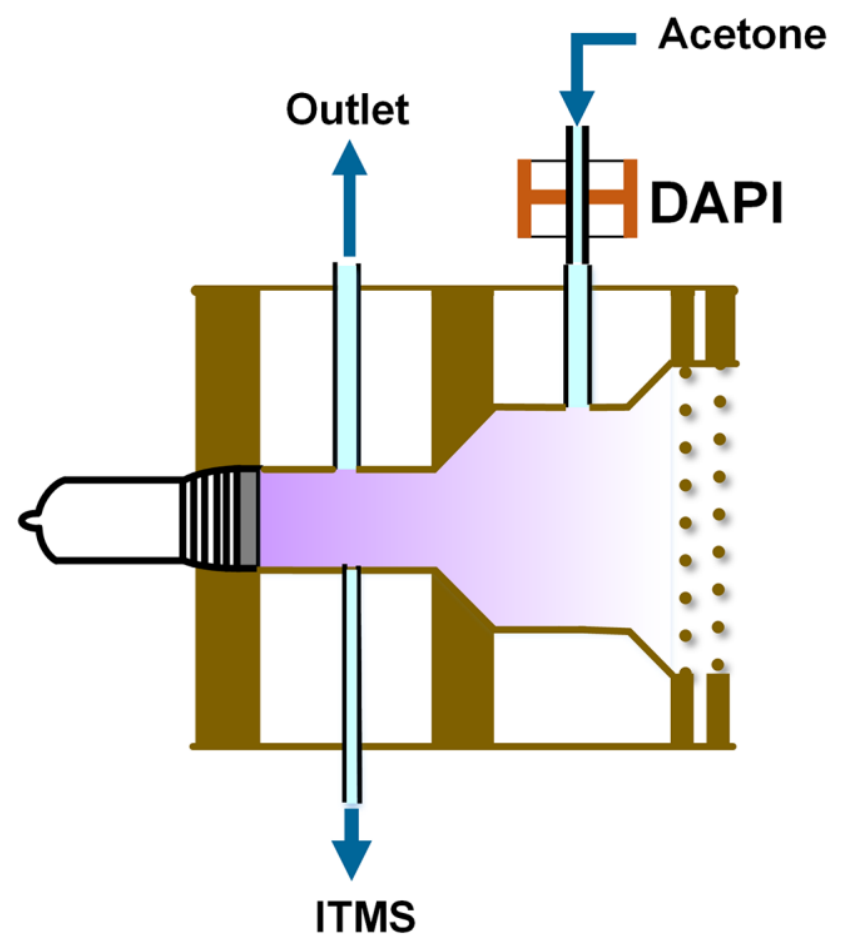

Figure S1. The schematic diagram of pulse injection structure.

Figure S1 presents the schematic diagram of pulse injection structure. A DAPI was used along with the sample inlet because the pulse width of DAPI was narrow and controllable. By adjusting the pulse width of the pinch valve and the flow rate of the drift gas, a minimum time-span of 0.5 $\mathrm{s}$ for acetone samples could be realized.

2. The Relative Position of Sampling Inlet of the ITMS System to the Axle Center of the IMS System.

The locations of the sampling inlet of the ITMS system is critical to the performances of both IMS system and ITMS system. Three positions of the ITMS system sampling inlet was investigated, as shown in Figure S2. 
Position A was near the outer surface of the ionization region, position B was near the inner surface of the ionization region, and position $\mathrm{C}$ was in the ionization region.

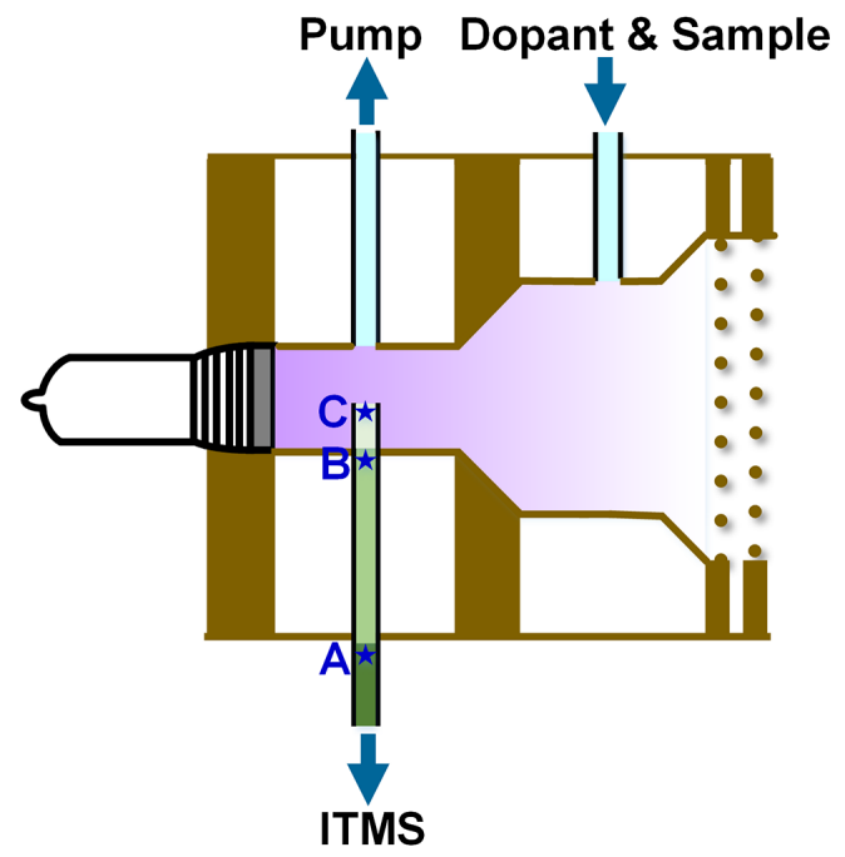

Figure S2. The relative position (marked as $\star$ ) of the sampling inlet of the ITMS system to the axial center of the IMS system. Position A was near the outer surface of the ionization region, position B was near the inner surface of the ionization region, and position $\mathrm{C}$ was near the axial center of the ionization region. 